\title{
Desafios do Ensino da Saúde Coletiva na Graduação dos Profissionais de Saúde
}

\author{
Heloniza O. G. Costa ${ }^{1}$ \\ Maria Ligia Rangel ${ }^{2}$
}

Resumo: O artigo busca caracterizar, em breves linhas, o campo da Saúde Coletiva e as tentativas de institucionalização de saberes e práticas que definem esse campo. Situa a emergência do campo e os desafios que se colocam para a incorporação do seu ensino na graduação dos profissionais de saúde, desde a década de 80. Discute as perspectivas do ensino da Saúde Coletiva na Graduação a partir do enfoque sobre o processo de trabalho em saúde e do fortalecimento de práticas desenvolvidas em parceria entre universidade, serviços de saúde e comunidade.

Palavras-chave: Formação de Recursos Humanos em Saúde; Saúde Coletiva - Graduação; Ensino de Profissionais de Saúde.

Summary: The article hopes to briefly characterize the Collective Health field and the attempts of institutionalizing knowledge and procedures involved. It gives an idea of how this field has grown and the challenges it has faced as it tries to be part of the health professionals training on the undergraduate level, since the $80 \mathrm{~s}$. It discusses the perspectives of the undergraduate Collective Health teaching based on the focus of the work process in health and on the strengthening of partnership procedures developed among university, health services and the community.

Keywords: Human Resources Qualification; Collective Health - Undergraduate level; Teaching of Health Professionals.

\footnotetext{
1 Professora da Faculdade de Enfermagem da UFBa. Diretora do Projeto UNI-Bahia.

2 Pesquisadora do Instituto de Saúde Coletiva da UFBA. Coordenadora da Secretaria Executiva da Rede UNIIDA.
} 


\section{Introdução}

A Saúde Coletiva caracteriza-se como um campo em constituição, com múltiplas concepções, objetos de conhecimento e práticas. Ao tomar como objeto o "coletivo", cuja noção tem conotações distintas, faz com que seus saberes e práticas sejam tributários de outros campos de conhecimento, além das ciências naturais, não subordinados ao estatuto de cientificidade desta, impossibilitando o ocultamento do caráter político da área (Donnangelo, 1983).

A emergência da Saúde Coletiva, enquanto campo de saber e práticas, defronta-se com a hegemonia da Clínica, que permeia a formação e a prática dos profissionais de saúde, marcada pela matriz flexneriana. A instrumentalização pela clínica, favorecendo a acumulação do capital no complexo médico-industrial e facilitando a geração de tecnologias materiais, teve uma relativa eficácia simbólica ou real e foi culturalmente assimilada pelos países ocidentais. Isso tornou o flexnerianismo a ideologia dominante no contexto supra-estrutural do modo de produção dos agentes das práticas de saúde, influenciando inclusive países que introduziram modificações significativas na organização dos serviços de saúde (Paim,1994).

A crise do modelo de atenção à saúde, sustentado pela matriz flexneriana de ensino, fez surgir vários movimentos reformistas ao interior, principalmente, das escolas médicas - medicina integral, medicina preventiva, medicina comunitária, integração docenteassistencial e medicina de família -, que influenciaram o conjunto das profissões de saúde. A maioria desses movimentos surgiu em países do primeiro mundo, difundindo-se aos países periféricos. Entretanto, esses movimentos não foram capazes de informar um projeto alternativo e crítico, consistente com uma análise científica da realidade. Contudo, no âmbito dos países latino-americanos a busca de alternativas para a crise estimulou e aprofundou o debate sobre o objeto da Saúde Pública, suas práticas e significados em distintas realidades. Assim, a formulação de um projeto crítico alternativo surge somente com o desenvolvimento da Medicina Social no Brasil, a partir da década de 70 , o qual forneceu conceitos e estratégias para a formação de Recursos Humanos em Saúde (Paim, 1992).

Do ponto de vista conceitual, a Saúde Coletiva surge apontando para a configuração de um novo paradigma para a. saúde, que procura imprimir um modo de pensar o processo saúde-doença como processo social e histórico, problematizando a organização das práticas de saúde com o propósito de introduzir mudanças, enfatizando a promoção da saúde, a prevenção de riscos, agravos e doenças a partir de estratégias de reorganização da vida social e não meramente através de medidas restritas aos serviços de saúde, articulando-se, assim, com estratégias mais amplas de construção da cidadania e de transformação da cultura sanitária e do modo de vida dos distintos grupos sociais.

Teixeira (1995) destaca que o campo da Saúde Coletiva tem abarcado três dimensões. Estas são, simultaneamente, áreas de investigação e de intervenção: estado de saúde da população, ou seja, condições de vida e saúde de grupos populacionais específicos e tendências gerais do ponto de vista epidemiológico, demográfico, socio-econômico, político e cultural; serviços de saúde, área que abrange desde o estudo do processo de trabalho em saúde (práticas de saúde), até a organização social dos serviços e a formulação e implementação de políticas de saúde; saber sobre saúde, que inclui desde estudos epistemológicos sobre produção de conhecimentos neste campo até relações entre o saber científico e as concepções e práticas populares de saúde.

O desenvolvimento da Saúde Coletiva, enquanto saber, compõe-se a partir da articulação de disciplinas básicas: a Epidemiolo- 
gia, a Administração é Planejamento de Saúde e as Ciências Sociais em Saúde, além de várias disciplinas auxiliares como a matemática, a informática, a demografia, a ecologia, a geografia, a sociologia, a antropologia, a história, as ciências políticas etc. (Teixeira, 1995). Enquanto prática, a Saúde Coletiva vem materializando uma ação social organizada no processo de Reforma Sanitária Brasileira (RSB) o qual, ao tempo em que conquista lugar social juridicamente definido, enfrenta resistências e obstáculos à sua implementação, que se fazem sentir tanto ao nível dos serviços de saúde quanto ao nível da formação de Recursos Humanos em Saúde (RHS).

A análise da atual conjuntura indica que a incorporação de saberes e práticas do campo da Saúde Coletiva no processo de graduação dos profissionais constitui um enorme desafio, à medida que o projeto neo-liberal se difunde e se fortalece. Neste contexto, em que o governo define como projeto assegurar a autonomia financeira e administrativa dos serviços sociais do Estado, o que se observa é que a Universidade, os serviços de saúde e os centros de pesquisa estão sendo estimulados a buscar auto-sustentação. Embora essa medida tenda a forçar a realização da missão dessas instituições com maior eficiência, ao mesmo tempo as conduz a se moldarem à lógica da economia de mercado.

Do ponto de vista do modelo de atenção, não se observa nenhum movimento no sentido de mudanças substanciais. A estrutura de financiamento, na sua essência, mantém-se intocada, embora apresente inovações que atualizam o seu modus operandi. Agregam-se, assim, programas com características focais, como o Programa de Saúde da Família (PSF), que demonstra claro viés complementar ao modelo hegemônico, na perspectiva de responder à grave crise social que afeta, especialmente, a saúde das populações de baixa renda. Por outro lado, observa-se que a preocupação com o desenvolvimento de RHS, particularmente a Graduação, tem per- manecido à margem das agendas políticas dos gestores do sistema de saúde e ausente das propostas centrais do Ministério da Educação. Dentre os primeiros, observa-se a preocupação, sobretudo, com a gestão de RHS, abordando aspectos tais como: mesa de negociação, terceirização versus estabilidade, salários, benefícios etc. As universidades, por sua vez, não estão discutindo a Graduação de RHS para o SUS, nem sendo chamadas a fazê-lo, mesmo quando se leva em conta programas estratégicos do governo com é o caso do PSF, para o qual não se tem definido o perfil profissional desejado.

Enquanto projeto contra-hegemônico, a RSB encontrou pouca penetração no tecido social, ao passo que o projeto hegemônico vai ao encontro de interesses individualistas da sociedade, em detrimento de um compromisso social mais amplo, fragilmente estabelecido entre os profissionais de saúde e entre segmentos do corpo docente das universidades. Além disso, o ensino da Saúde Coletiva na Graduação depara-se com problemas próprios da sua dinâmica interna, tais como: as práticas/trabalho que não são assumidas como locus privilegiado do processo ensino-aprendizagem; metodologias que não instrumentalizam os alunos para a produção de conhecimentos e o desenvolvimento da capacidade crítica e criativa, além de não favorecer a criação de atitudes construtivas e participativas. Estas distorções são possibilitadas pela desarticulação de conteúdos; pela excessiva fragmentação das disciplinas e dos departamentos; pelo uso de metodologias pedagógicas inadequadas ao processo de aprendizagem; pelo conservadorismo docente; pela inadequação de conteúdos às necessidades de saúde da população e pela desarticulação com os serviços de saúde e com a comunidade, entre outros.

Daí, o desafio que se apresenta refere-se à possibilidade de ampliação dos espaços do projeto contra-hegemônico, tanto ao nível das universidades quanto nos serviços de saúde 
e na sociedade civil. Assim, na vida cotidiana dos serviços de saúde, trata-se de obter mudanças da organização do trabalho no interior dos serviços de saúde. No âmbito das universidades, no que se refere à Graduação, trata-se de obter a adesão dos docentes a novas formas/metodologias que permitam aos alunos a apropriação do conhecimento e o desenvolvimento de novas habilidades, de modo a poder refletir o seu lugar na sociedade a partir do seu lugar no trabaIho, aprendendo um fazer crítico e criativo. No que tange à sociedade civil, trata-se de ampliar as práticas de participação social, seja ao nível da gestão de serviços de saúde como também das universidades.

A articulação da Universidade com os serviços de saúde e a comunidade permite, potencialmente, o desencadeamento sincrônico e simultâneo de capacitação pedagógica de docentes, de desenvolvimento de programas de educação permanente para profissionais de saúde e do desenvolvimento de lideranças comunitárias na direção das mudanças pretendidas, ampliando, desse modo, a difusão das concepções e reorientando as práticas de saúde no interior das organizações.

Pretende-se assim, levantar alguns pontos para a reflexão acerca dos limites e possibilidades da restruturação da Graduação dos profissionais de saúde na perspectiva da Saúde Coletiva.

\section{O Ensino da Saúde Coletiva na Graduação}

O ensino da Saúde Coletiva se confunde com a história e constituição desse campo (Nunes, 1996). O ensino de Saúde Coletiva originou-se nos Departamentos de Medicina Preventiva (DMP) cuja criação é reflexo, nas escolas médicas, do processo de reformas que buscaram enfrentar a crise do modelo de atenção à saúde médico-privatista, caracteri- zada pela crescente incorporação tecnológica e elevados custos. Analisando as questões referentes ao desenvolvimento de RHS no Brasil, Paim (1994) observa que a reformulação do ensino das profissões de saúde, sobretudo da formação médica, tem sido uma preocupação dos órgãos formadores em geral, inclusive do MEC. No entanto, a direcionalidade dessas mudanças tem sido múltipla, apontando para a recuperação do caráter liberal da profissão ou para a atualização da formação frente às modificações na organização dos serviços de saúde, pouco voltadas às necessidades de saúde e direitos dos cidadãos (Paim, 1994).

A análise dos movimentos reformistas permite constatar que estes provocaram mudanças no Sistema de Saúde no Brasil, com algumas repercussões nos modelos de ensino nos cursos superiores na área da saúde. Algumas dessas mudanças, a exemplo das atividades extra-muro, impulsionadas pela perspectiva da atenção primária à saúde, proposta pela Conferência de Alma Ata (1978), geraram insatisfação em alguns Departamentos de Medicina Preventiva e na própria Organização Panamericana da Saúde (OPAS) com relação aos limites dos projetos extramuro em se contrapor ao modelo flexneriano de ensino, conduzindo à emergência da proposta de Integração Docente Assistencial IDA (Marsiglia et al., 1996).

$\mathrm{Na}$ década de 80, a IDA buscava atender expectativas de promover mudanças profundas nos currículos das faculdades da área de saúde. Em 1981, o MEC elaborou uma publicação onde definia a IDA, e apelava às universidades a aceitarem o desafio da proposta, o que exigiria modificações conceituais, estruturais e metodológicas profundas, contrariando o clássico conceito ensino-pesquisa-extensão, na busca de novas relações universidade-prestadores de serviços.

Em 1987, a portaria interministerial MEC/ MPAS estabelece critérios e parâmetros para aplicação de um Índice de Valorização de 
Desempenho para hospitais universitários e de ensino (IAV-EM - Índice de Adicional de Valorização e Capacitação Extra-Mural), e, em 1988, o MEC aprova e divulga nova proposta - Plano UNISIS - Integração das Universidades no Sistema de Saúde, em consonância com os princípios do SUDS. Isso significava que as atividades de ensino das universidades se estenderiam a todos os profissionais da rede para a promoção da educação continuada (reciclagem, especialização, aprimoramento etc.). Entendia-se que qualquer serviço do Sistema de Saúde seria formalmente reconhecido pela Universidade como local de ensino, e não só o hospital-escola. As atividades docentes extra-muros deveriam ser de natureza curricular, obrigatória para todos os alunos do curso regular.

A análise dos 10 anos de IDA no Brasil revela que: as experiências se concentraram nos DMP; algumas vezes com participação da pediatria e, mais raramente, da tocoginecologia. A maioria das experiências IDA-Brasil é desenvolvida em universidades federais e estaduais, localizadas na região Sudeste do país, apoiadas originalmente pela Fundação Kellogg, e concentradas nos cursos de medicina, enfermagem e odontologia (Marsiglia, 1995).

De acordo com Paim (1994), tais experiências, ao tentarem interferir nas práticas de saúde, se depararam com distintas visões e interesses que competem entre si, visando moldar a definição do perfil dos profissionais. Os estudos e reflexões sobre as tentativas de introduzir mudanças na formação dos profissionais de saúde apontam sempre o dilema dessas propostas para se institucionalizarem e redirecionarem, de fato, o processo de formação dos profissionais. Ainda segundo esse Autor, nas décadas de 70/80 houve uma significativa produção teórica da Medicina Social relacionada aos cursos de Pós-Graduação, numa estreita relação com os movimentos sociais presentes na conjuntura. De todo modo, a maior expressão do desenvol- vimento da Saúde Coletiva limitou-se à PósGraduação, através dos cursos de mestrado, da especialização em Saúde Pública, dos programas de residência em Medicina Preventiva e Social.

No âmbito da Graduação, surgiu, na década de 80, estimulada pela ABRASCO e pela Associação Brasileira de Ensino Médico (ABEM), uma proposta de conteúdo programático mínimo para o ensino da Saúde Coletiva, centrado nas ciências sociais, epidemiologia, administração e organização de serviços. Esta foi elaborada a partir da constatação da diversidade de conteúdos programáticos e de metodologias de ensino, e da heterogeneidade da bibliografia utilizada nesta área. No entanto, apesar da sua incorporação e avaliação por alguns departamentos de Medicina Preventiva e Social, não se dispõe de estudos avaliativos, seja na área médica, seja nas demais áreas (Paim, 1994).

\section{Perspectivas do Ensino da Saúde Coletiva na Formação dos Profissionais de Saúde}

A necessidade de imprimir uma nova lógica ao processo de ensino/aprendizagem, na perspectiva da inserção da Saúde Coletiva no processo de formação dos profissionais de saúde, implica enfrentar a rigidez dos modelos praticados e redesenhar a estrutura curricular e modelos pedagógicos, em todos os âmbitos.

O desenvolvimento conceitual do campo da Saúde Coletiva aponta a abordagem das tecnologias em saúde como a de maior potencial para a reflexão e prática da Saúcle Coletiva. Segundo Ayres (1996), isso se observa no pensamento de Gonçalves "quando propōe a tecnologia como trabalho já em operaçāo, e, como tal, efetiva construção da sociedade", superando a concepção de tecnologia como "instrumento dado a priori, fundado num saber objetivo e universal e cujo suces- 
so ou fracasso dependeria apenas de uma boa ou má economia de meios e fins" (Ayres, 1996).

Gonçalves identifica o processo de trabalho em saúde como espaço privilegiado para a efetivação de mudanças, dada a possibilidade de transformação dos objetos de trabalho, à medida que se alteram os meios e as relações de trabalho, que constituem o substrato da organização das práticas (Paim, 1996). No momento em que tecnologia é pensada como organização do trabalho, o conceito de doença ganha um significado distinto daquele que lhe é atribuído normalmente pelo senso comum. Para Gonçalves, "a doença não é tão somente objeto do trabalho em saúde mas é igualmente e simultaneamente, instrumento desse trabalho." A própria concepção da doença vai ser produto e produtor de certas formas e condições de aproximação às questões da saúde - o conceito de doença não está dado $a$ priori. O desafio que se impõe é o de apreender e operacionalizar a concepção de saúde enquanto objeto e instrumento orientador do trabalho. Saúde entendida ou representada, não como ausência de doença, mas relacionada à qualidade de vida, tal como presente nas estratégias de Vigilância da Saúde, cuja ênfase recai sobre a promoção da saúde (Mendes, 1993).

Essa reconstrução deixa clara a situação estratégica da ciência nas práticas de saúde. Portanto, a Saúde Coletiva, a requerer novas formas de organizaçào do trabalho, exige novos saberes orientadores dessa prática (Ayres, 1996), abrindo-se uma perspectiva interdisciplinar ou mesmo transdisciplinar.

Face aos problemas que enfrenta, a inserção da Saúde Coletiva na graduação, significa mais que a introdução de um campo de conhecimento e prática. Exige, em decorrência da concepção de saúde e da prática profissional que adota, uma mudança no processo de formação dos profissionais de saúde como um todo.
As experiências de ensino da Saúde Coletiva na Graduação, enquanto introdução de inovações através de uma única disciplina, têm se defrontado com dificuldades estruturais que não se resolvem no âmbito disciplinar, e nem mesmo no âmbito acadêmico, isoladamente. Isso pode ser visto na experiência do DMP/UFBa com relação à disciplina "Saúde Pública e Medidas de Profilaxia", a qual buscou aproximar os alunos dos principais problemas de saúde da população a partir do enfoque da dimensão coletiva do processo saúde-doença e das técnicas e estratégias existentes para o controle destes problemas. Apresentou resultados positivos no sentido de ter atenuado as resistências dos alunos quando compreenderam a utilidade do saber adquirido para a análise da situação de saúde e seus determinantes, bem como das formas de intervenção e definição do processo de gestão (Silva et al.,1993). Do mesmo modo, o ensino da disciplina "Introdução à Medicina Social", desse mesmo DMP, incluiu a produção de conhecimentos sobre as relações entre processo saúde/doença e o modo de vida de grupos populacionais específicos e as respostas socialmente organizadas para o enfrentamento dos problemas de saúde com base na ação estatal e/ou na ação das organizações políticas, profissionais, sindicais e populares. Essa disciplina despertou o interesse dos alunos na ampliação do leque de discussões acerca de aspectos históricos, políticos e sociais na prática médica (Teixeira et al., 1994). A experiência do DMP/UFBa, com o ensino da Medicina Preventiva em Centros de Saúde ao longo de 3 anos, numa proposta em parceria com a Secretaria de Saúde do Estado da Bahia (SESAB), melhorou a coordenação docente, o material didático, a receptividade dos alunos, a relação professor-aluno, e propiciou a modernização pedagógica. Contudo, essas experiências se depararam com problemas estruturais tais como dificuldades para reorganização das práticas de saúde ao nivel dos serviços de 
saúde que serviram de cenário para o ensino, cuja lógica é predominantemente aquela dos modelos assistenciais voltados para o atendimento da demanda espontânea. Ou seja, buscava-se ensinar nos serviços uma prática inexistente nesses serviços, onde os profissionais permaneciam por pouco tempo, implicando descontinuidade da colaboração com as atividades de ensino, baixa demanda, falta de apoio diagnóstico, falta de insumos e desativação de unidades em períodos de campanhas, entre outros (Teixeira et al., 1984).

Uma alternativa mais ampliada que se identifica no cenário, no momento atual, enquanto proposta de redirecionamento do ensino das profissões de saúde a partir do referencial da Saúde Coletiva, está contida, de algum modo, no Programa $\mathrm{UNI}^{3}$, na medida em que este assume, como eixo central, a construção da parceria ensino-serviço-comunidade, para imprimir mudanças na formação dos profissionais de saúde, na reorganização das práticas de saúde e na forma de participação social, definindo como estratégia fundamental a articulação do mundo do ensino ao mundo do trabalho.

Ainda que na forma de esforços localizados, os projetos UNI têm buscado o enfrentamento de problemas técnico-pedagógicos, tais como: definição de objetivos educacionais; capacitação pedagógica de docentes; uso de tecnologias ativas no processo ensino-aprendizagem; diversificação dos cenários de práticas; valorização do processo de trabalho como locus privilegiado do aprendizado; superação dos espaços disciplinares existentes nos currículos, favorecendo a produção de conhecimento interdisciplinar; de-

(3) O Programa UNI - Uma Nova Inicjativa na educação dos profissionais de Saúde: União com a Comunidade, foi proposto no início da década de 90 pela Fundação $W$. Kellogg, e vem sendo desenvolvido por 23 universidades na América Latina, sendo 6 no Brasil. Envolve necessariamente universidades, serviços de saúde e comunidade. senvolvimento de práticas multiprofissionais; articulação entre o ciclo básico e o ciclo profissionalizante; responsabilização das universidades com a qualificação dos profissionais de serviços de saúde.

Mesmo parciais, as práticas dos projetos UNI, ao explorarem os limites e possibilidades de cada conjuntura, podem introduzir inovações, progressivamente, mediante a produção de fatos nas escolas, nos serviços e comunidades, que possibilitem o acúmulo de experiências e saldos organizativos, que articulados a movimentos sociais possam influir na obtenção das mudanças desejadas. Buscase a ampliação dos direitos sociais e a conquista da cidadania.

As propostas de reforma do modelo de ensino e de atenção à saúde, trazidas pelos UNI, embora enfrentando conjunturas desfavoráveis, têm ampliado o debate sobre formação e desenvolvimento de RHS e políticas de saúde entre professores, estudantes, profissionais de serviço de saúde e população. Isso tem resultado no crescimento do processo participativo no interior das instituições de ensino, num movimento sinérgico à luta pela democratização da saúde e da educação brasileiras.

A perspectiva é que projetos como os UNI e outras experiências que têm adotado a mesma direcionalidade, neste momento compondo a Rede UNIIDA, se transformem em um ator político capaz de imprimir um movimento que contribua para alterar a correlação de forças institucionais, adequando a formação dos profissionais às necessidades de saúde da população. Desse modo, a Rede UNIIDA propõe mudanças nas relações do ensino com o trabalho em saúde, problematizando as instituições de ensino no processo de redefinição dos serviços de saúde e de implementação do SUS.

Um outro movimento observado referese à perspectiva expansionista do ensino de Saúde Coletiva na graduação adotada pelo Instituto de Saúde Coletiva - ISC/UFBa, 
que envolve unidades responsáveis pela formação profissional na área da saúde (Medicina, Nutrição, Odontologia, Farmácia, Medicina Veterinária), "com o propósito de fortalecer um componente geral e comum ao perfil educacional de todos os profissionais de saúde, cabendo a cada unidade desenvolver conteúdos e habilidades específicas a cada profissão, de acordo com o lugar que ocupa na divisāo técnica e social do trabatho em saúde" (Teixeira, 1995).

Adota-se como eixo do ensino de Saúde Coletiva na graduação "a prática epidemiológica em serviços, a implementação de ações de Vigilância da Saúde e o desenvolvimento de ações de Promoção da Saúde junto a grupos sociais organizados e população em geral, privilegiando a inserção dos estudantes na prática, tendo como espaço de atuação o Distrito Sanitário" (Teixeira, 1995). Busca-se, também, a articulação do ensino de Graduação com o processo de municipalização e distritalização.

\section{Considerações Finais}

$\mathrm{Na}$ atualidade, vale pensar com Demo (1996) o significado da educação com qualidade. Ou seja, pensar o processo formativo como uma contínua produção de conhecimen- to com qualidade formal e política ${ }^{4}$. Esta qualidade implica, também, formar competência capaz de preparar para a vida e dar conta de novos desafios. Estes desafios, no campo da formação dos profissionais de saúde, exigem um trabalho que transcende a ação puramente técnica do corpo docente, de mudanças de conteúdos programáticos e grade curricular. Requerem esforços para criar viabilidade de construção de um novo processo ensino-aprendizagem em consonância com a cidadania.

Portanto, os limites e possibilidades da inserção da Saúde Coletiva no ensino das profissões de saúde não estão dados $a$ priori. Os avanços e retrocessos dependerão da capacidade de mobilização e articulação das forças interessadas no interior das universidades com outros segmentos da sociedade. A difusão de suas concepções e práticas deve se dar amplamente, no sentido de alcançar maior capilaridade social e, portanto, ampliar seu potencial de influir. nos desenhos e conteúdos curriculares, no âmbito interno, e nas políticas educacionais das profissões de saúde, no âmbito social mais amplo. Nesse sentido a parceria com os serviços de saúde dirigidos à comunidade constitui estratégia privilegiada para conferir sustentabilidade a tal projeto.

\footnotetext{
(6) Acerca da qualidade formal, o Autor destaca que esta é mais fácil de ser manejada que a política. A primeira "codifica-se em expressōes metodológicas que admitem consensos, como tessitura lógica, uso bibliográfico, argumentação, condução da hipótese de trabalho (...)". A qualidade política "(...) está muito mais na maneira de ser, ética, engajada, inovadora, do que em manifestaçōes estereotipadas, que facilitam o tratamento metódico, mas podem estar muito distantes da realidade" (Demo, 1996:112).
} 